\title{
Eosinophilic Esophagitis: Asthma of the Esophagus?
}

\author{
J. Christian Virchow \\ Department of Pneumology, Intensive Care Medicine, Klinikum der Universität Rostock, Rostock, Germany
}

\section{Key Words}

Asthma · Pathogenesis · Eosinophils · Esophagitis ·

Similarities

\begin{abstract}
The question whether eosinophilic esophagitis (EoE) might be an 'asthma of the esophagus' is reasonable. There are a number of similarities between the two diseases: EoE and asthma, as well as other atopic diseases, are frequently associated and have a number of similarities in their pathogenesis. Thus, investigating differences and similarities between the diseases might be a worthwhile endeavor. Both EoE and asthma are chronic immune-mediated conditions characterized by inflammatory changes in the mucosa and submucosa with a characteristic and diagnostic infiltration of eosinophils. They result in organ dysfunction with considerable morbidity and (in the case of asthma) even mortality. Asthma and EoE affect all ages, but frequently start in childhood or adolescence. While asthma has seen a large increase in its prevalence in the past 50 years, EoE was first described in the 1970s. Since then the frequency of the diagnosis of EoE has increased significantly. The prevalence for both diseases seems to be highest in the Western world. In contrast to asth$\mathrm{ma}$, where females are more often affected, EoE is more frequent in males. Asthma in children, however, is also more common in boys, but this changes after puberty. EoE is frequently associated with asthma, and up to $80 \%$ of patients
\end{abstract}

with EoE are atopic, similar to childhood asthma. Adult-onset asthma is not necessarily associated with atopy (termed intrinsic asthma) and similar observations have been made for EoE. Endoscopically, asthmatic airway mucosa as well as esophageal mucosa in EoE can appear normal, and biopsies are required for diagnosis. Long-standing disease in asthma has been associated with 'remodeling' compared to predominantly reversible inflammatory changes early in the course of the disease. Similar observations have been made in EoE. Toxic proteins derived from eosinophils such as major basic protein, eosinophil-derived neurotoxin and eosinophil cationic protein can be found in the mucosa of both diseases, which are also characterized by a thickening of the lamina propria or basement membrane, respectively. Despite these histologic and immunochemistry findings, asthma as well as EoE remain clinical diagnoses, and diagnosing either condition can be challenging. Therapeutically, both diseases respond well to corticosteroids. Ironically, corticosteroids for inhalation are deliberately swallowed in EoE to reach the esophageal mucosa. Allergen/food avoidance can improve symptoms in asthma and EoE. Taken together, allergic asthma and EoE have a number of common features which make a common pathogenesis manifested in different organs for reasons not yet fully understood likely. Combining allergological research with gastroenterologic and pneumologic expertise with a focus on similarities between these diseases might be a way forward.

(c) 2014 S. Karger AG, Basel

\section{KARGER}

E-Mail karger@karger.com

www.karger.com/ddi
C 2014 S. Karger AG, Basel

0257-2753/14/0322-0054\$39.50/0
J. Christian Virchow

Department of Pneumology, Klinikum Universität Rostock

Ernst-Heydemann-Strasse 6

DE-18057 Rostock (Germany)

E-Mail j.c.virchow@ med.uni-rostock.de 


\section{Introduction}

While bronchial asthma has been recognized for many decades with a sharp increase in prevalence in the last 30 years, thus gaining the attention of specialists as well as general practitioners, eosinophilic esophagitis (EoE) has only recently gained wider attention from a mainly specialized audience of pediatricians and gastroenterologists. Guidelines which address both diseases together do not exist. Yet, a number of similarities between the diseases make it worthwhile to compare these diseases according to their pathophysiology and pathogenesis.

Asthma is a disease defined by clinical, (patho)physiologic and pathologic characteristics. Its clinical history includes episodic dyspnea that frequently occurs at night and is often accompanied by coughing. Wheezing is the most common clinical sign, but in some patients coughing can be the only symptom. Pathophysiologically, patients with asthma have (episodic) airflow obstruction, expiratory flow limitation and a characteristic bronchial hyperresponsiveness to nonspecific stimuli such as cold air, second-hand smoke, fumes, dusts, etc. These findings are caused by inflammatory changes in the airways which include many different inflammatory cells and mediators, but also structural cells of the airways. Its pathogenesis is unclear: genetic as well as environmental influences have been described with no clear pattern of inheritance.

\section{Similarities between Asthma and EoE}

Both asthma and EoE are chronic immune-mediated and most likely antigen/allergen-driven conditions. They are characterized by inflammatory changes in the mucosa and submucosa with a typical infiltration by eosinophils that are diagnostic features of both diseases. This inflammation is believed to be the cause of the organ dysfunction observed in asthma as well as EoE that results in considerable morbidity and (in the case of asthma) even mortality. Both diseases appear to occur preferentially on an allergic background, but atopy is not a prerequisite for either illness.

Asthma and EoE can present with variable symptomatology. There are no specific or exclusive symptoms to either disease; therefore, the delay in diagnosis can be considerable, with EoE having an even larger time span between occurrence of symptoms and diagnosis [1]. Children with either condition commonly have a failure to thrive [2].

EoE: Asthma of the Esophagus?
A genetic background has been proposed in both EoE and asthma, but direct proof is still missing. In asthma the genetic basis has been investigated in many studies and the genetic determinants of allergic asthma are, at best, multifaceted [3], while in EoE a genetic underpinning has been suspected based on SNPs for eotaxin, TSLP and the TSLP receptor [4-6]. Still asthma as well as EoE remain clinical diagnoses, and response to treatment is a valuable diagnostic tool in both diseases.

Asthma and EoE can affect patients at all ages, but both diseases often start during childhood and adolescence, with the highest prevalence found in the Western world. For asthma, several hypotheses have been put forward to explain the recent increase in prevalence as well as the fact that asthma is preferentially associated with features of the Western lifestyle. The most accepted hypothesis assumes a lack of exposure of the immune system of young children and/or their mothers to ubiquitous microbial antigens and/or unpasteurized cow's milk, and a resulting failure of the adaptive immune system to differentiate between potentially harmful and harmless antigens $[7,8]$.

Due to the atopic background of many patients with asthma or EoE, seasonal worsening of symptoms is common $[9,10]$. The fact that atopy is a systemic condition with the potential to manifest itself with disease-specific symptoms in several organs is also reflected by the fact that EoE is frequently associated with other atopic conditions such as asthma, allergic rhinitis, atopic dermatitis and food allergies [11]. Asthma is often preceded by food allergies in children, and similarly food allergies seem to be driving EoE in children, while in adults aeroallergens are more often associated with worsening of asthma as well as EoE [11]. Allergen avoidance often improves symptoms in both diseases, but it is not curative in either condition $[12,13]$. However, adult onset of both diseases is not necessarily associated with atopy for which the term intrinsic asthma has been used in asthma [14].

\section{Comparative Pathology: Similarities and Differences}

Asthma and EoE can have macroscopically normal mucosa (although chronic EoE causes rather characteristic appearances of the esophagus), which, however, have dense infiltrates of eosinophils (including eosinophil microabscesses), mast cells and lymphocytes. The accumulation of mast cells in EoE has resulted in the suggestions to rename and/or redefine this disease. While mast cells in asthma have been associated with the early response to allergens, their role in the chronic manifestation of asth- 
ma remains unclear. Both asthma and EoE demonstrate a Th2 cell signature with elevated Th2 cytokine levels or signals. Toxic proteins from eosinophils have been shown to be deposited in the bronchial [15] or esophageal mucosa [16] in asthma and EoE, respectively. Elevated concentrations of eosinophil cationic proteins have also been associated with parameters of airflow limitation in asthma [17]. Long-standing disease in asthma as well as EoE is associated with an ill-defined 'remodeling'. This includes structural changes to the mucosa but more importantly to the submucosa, which can contribute to organ dysfunction as well as to the lack of therapeutic responses in chronic disease. While thickening of the basement membrane has been consistently reported for bronchial asthma, fibrosis of the lamina propria seems to be typical in EoE. While these latter changes are likely to contribute to treatment-refractory disease, the inflammation with eosinophils is predictive of a good response to treatment in the majority of cases of asthma or EoE. On the other hand, there is quick recrudescence of symptoms after cessation of allergen avoidance or steroid therapy.

In asthma, the degree of airflow obstruction as well as the response to therapy has been associated with eosinophil activation as well as the concentrations of toxic cationic proteins derived from eosinophils [17], and there is an inverse relationship between pulmonary function and indices of asthmatic inflammation such as activated $\mathrm{T}$ lymphocytes, eosinophil numbers and Th2 cytokines such as IL-5 [18].

Elevated numbers of mast cells with features of mast cell activation have been demonstrated in both asthma and EoE. In asthma, mast cells could potentially contribute chronically to organ dysfunction. Yet, histamine that is released from mast cells might not be the most important mediator since antihistamines are clinically useless in asthma and EoE. On the other hand, mast cells have been shown to release a number of cytokines, proteases and bioactive compounds that could contribute to chronic inflammation in both diseases [19]. Another striking similarity of asthma and EoE is the fact that the vast majority of these mast cells are carboxypeptidase A3 and tryptase high but chymase low [20, 21]. In EoE, mast cell-derived TGF- $\beta$ has been shown to cause smooth muscle contraction [22], but TGF- $\beta$ has also been reported to be associated with eosinophils in EoE [23] making the relative contributions of either cell population unclear. In asthma, elevated concentrations of TGF- $\beta$ have been measured endobronchially [24], and a recent publication has described TGF- $\beta$ - and MMP-2-dependent collagen-1 production as potential causes for airway remodeling [25].
Among the differences in the epidemiology, pathology and pathogenesis of the two diseases, it is noteworthy that asthma has had an almost epidemic increase in the past 50 years. The cause of this increase is unclear. EoE, on the contrary, was first described in the 1970s. While more boys than girls are affected by asthma during childhood, this ratio appears to change after puberty with a male:female ratio of approximately $2: 3$ in adulthood. In EoE, males have been reported to be more often affected than females [11].

The histopathologic changes of eosinophilic microabscesses observed in EoE [26] have not been reported for asthma.

\section{Differential Diagnosis}

The diagnosis of asthma in children is complicated by the similar clinical appearance of viral respiratory infections as well as recurrent bronchitis in adults. Furthermore, smoking-associated airway damage and resulting chronic bronchitis as well as recurrent aspiration of gastric content in symptomatic and asymptomatic gastroesophageal reflux can mimic or complicate asthma. In EoE, reflux-induced eosinophilia of the esophagus is a major differential diagnosis, and it has been proposed that proton pump inhibitor therapy should be tried before making the diagnosis of EoE.

\section{Response to Treatment}

\section{Topical Steroids}

Both bronchial asthma and EoE respond to systemic and possibly even better to local glucocorticosteroid therapy.

\section{Omalizumab}

While asthma has been shown to respond to anti-IgE treatment in a number of studies which showed a reduction in the number of exacerbations [27], the response of EoE to this form of treatment has not been systematically investigated, and positive responses are limited to case reports [28]. While there was some symptomatic improvement in these patients, the number of eosinophils did not change. However, recent data in asthma has challenged the concept that a reduction of circulating IgE antibodies alone are sufficient to explain the observed treatment response [29] since positive responses to anti-IgE therapy with omalizumab have also been reported in socalled intrinsic asthma. It has been hypothesized that ei-
56

Dig Dis 2014;32:54-60

DOI: $10.1159 / 000357010$
Virchow 
ther the reduction of locally produced IgE or interference with the IgE receptor on dendritic cells might cause the observed effects in intrinsic asthma.

\section{Interleukin-13}

IL-13 has been measured in elevated concentrations in patients with asthma, and allergen challenge further increases local IL-13 production in asthma [30]. Recent studies in asthma have shown that anti-IL-13 treatment, especially in patients with a high IL-13 signature, as measured by periostin expression, can improve pulmonary function [31]. In EoE, an IL-13-induced EoE-specific transcriptome has been described which has been associated with an upregulation of eotaxin and periostin [32]. Furthermore, an IL-13-dependent decrease in filaggrin and involucrin has been reported for EoE [33], but this has not yet been investigated in biopsies from patients with asthma. Recent studies (in mice, however) have suggested that the esophageal eosinophilia in IL-13-deficient mice is IL-13 independent while the pulmonary eosinophilia is IL-13 dependent [34].

Although early studies using anti-IL-5 antibodies in asthma have not been able to show any relevant clinical response (most likely due to the selection of wrong endpoints) [35], a clinical improvement on anti-IL-5 treatment has been reported for EoE. However, there was no significant or meaningful difference compared to placebo in these investigations [36]. In asthma, recent studies using anti-IL-5 antibodies have shown a reduction in exacerbations [37] and even an improvement in lung function [38]. In EoE, a large symptomatic improvement has been reported after several doses of reslizumab. Again, there was a big placebo effect on EoE symptoms which led to a nonsignificant improvement using this anti-IL-5 strategy.

\section{Phenotypes and Endotypes}

Several phenotypes have been suggested for asthma, based on symptoms and other clinical observations. However, until today most of these phenotypes have been insufficiently separated from parameters of severity. While there are two clearly different clinical entities or phenotypes, namely allergic asthma and intrinsic asthma, it is unclear if other clinical parameters indeed define a separate phenotype. Based on sputum cytology, asthma can be further separated into eosinophilic, neutrophilic and paucigranulocytic phenotypes. These differences, however, might not reflect the true inflammation underlying these conditions. Furthermore, there is evidence that these 'phenotypes' are far from being stable. Many patients with so-called sputum-defined neutrophilic asthma will devel-
Table 1. Clinical and histological features of allergic and intrinsic asthma [48]

\begin{tabular}{lll}
\hline & Allergic asthma & $\begin{array}{l}\text { Intrinsic } \\
\text { asthma }\end{array}$ \\
\hline Onset & $<30$ years & $>40$ years \\
Family history & + & - \\
Other atopic & & \\
$\quad$ manifestations & + & - \\
Seasonal symptoms & based on sensitization & - \\
Perennial symptoms & based on sensitization & + \\
Asthma attacks & + & - \\
Skin prick tests & + & - \\
Total IgE & elevated & normal \\
Specific IgE & detectable & - \\
Eosinophilia & + & ++ \\
Sputum eosinophilia & + & ++ \\
Chronic sinusitis & - & + \\
Nasal/sinus polyps & - & + \\
Aspirin-induced asthma & rarely & + \\
Response to therapy & + & - \\
Steroid-free intervals & + & - \\
Steroid requirement & - & + \\
\hline
\end{tabular}

op sputum eosinophilia when followed longitudinally [39]. Therefore, the diagnosis of asthma should be challenged in patients with persistent neutrophilia. Recently, ill-defined basic mechanisms which are still vaguely defined but which might cause the discussed phenotypes have been proposed. This concept, however, has yet to prove such basic differences [40] and therefore the concept of endotypes is still reminiscent to the tale of 'The Emperor's New Clothes'. Whether there are forms of EoE which do not necessarily contain the number of eosinophils required to support or confirm the diagnosis is unclear. In asthma, allergic and intrinsic asthma can be separated based on a number of clinical and histological features (table 1).

In EoE, a nonatopic form has also been identified, but its pathogenetic background and differences to the allergic type are still largely unclear. In contrast to allergic asthma, patients with intrinsic asthma have been shown to have an IL-2-high, IL-4-low and CD23-low background, but both are characterized by elevated IL-5 concentrations. In contrast to allergic asthma, elevated numbers of activated, IL-2R-, HLA-DR-, and VLA-1-positive CD8-T cells have been described in intrinsic asthma [41], which has not been seen in allergic asthma, suggesting that patients with intrinsic asthma might have a chronic antigen exposure, possibly due to an endogenous antigen [41]. Similar mechanisms might be operating in nonallergic forms of EoE, but this has not been studied. 
Some novel aspects in the pathogenesis of asthma that might also be of interest to EoE are the demonstration that upon antigen/allergen challenge there is a large increase in the number of dendritic cells, both of the myeloid as well as the plasmacytoid phenotype [42]. Dendritic cells play an important role in the regulation of inflammation, and targeting dendritic cells might have a therapeutic benefit. This requires further exploration and therefore similar investigations might be of interest to better understand the immune regulation in EoE, too.

EoE has been associated with esophageal dysmotility, which has been attributed to structural changes in the submucosa leading to impaired movements of the esophageal wall. In asthma, airway dysfunction which leads to airway narrowing has been attributed in part to airway inflammation and also in part to structural as well as neurogenic changes [43]. Together, these changes have been associated with the characteristic bronchial hyperresponsiveness in asthma. Recently, the demonstration of increased concentrations of nerve growth factors has lent support to the hypothesis that neuronal changes might be intimately involved in the characteristic bronchial hyperresponsiveness in these patients. Upon allergen challenge, nerve growth factors with their potential to modulate the autonomous nervous system in the lungs increase considerably $18 \mathrm{~h}$ following allergen challenge [44]. While this alone might be sufficient to change autonomic nerve functions in the airways, it has also been shown that endobronchial, but not peripheral blood, eosinophils' survival is markedly enhanced in the presence of these nerve growth factors [45]. This suggests that allergen exposure not only leads to the release of factors which can influence the autonomic nervous system of the lungs but also enhances eosinophil viability and thus endobronchial inflammation. Endobronchial eosinophils express nerve growth factor receptors [45], but the regulation of these receptors remains unclear. Thus, neurotrophin concentrations increase in the airways of patients with asthma following allergen challenge and can activate endobronchial eosinophils that express receptors for neurotrophins. At present it can only be speculated if similar changes in the esophageal mucosa might contribute to the esophageal dysfunction that many affected patients have reported.

Finally, one unsolved puzzle in the understanding of asthma is the fact that asthma can progress even in the absence of allergen exposure. This has been explained with the observation that asthma is characterized by a chronic inflammation with some features of an autoimmune disease. This would be in line with recent hypoth- eses claiming an inverse relationship between the observed reduction in infectious diseases such as tuberculosis, measles, mumps, etc., and the inverse increase in so-called autoimmune disorders such as multiple sclerosis, Crohn's disease and type 1 diabetes. Investigations into possible autoimmune phenomena have been largely restricted to humoral immunology with no clear results. In the cellular compartment in asthma, however, in addition to the well-known increase in Th2 lymphocyte-associated changes, there is an increase in cytotoxic cells with cytotoxic granzyme B release into the airways [46]. This increase in granzyme B is regulated by IL-3 and there is a correlation between granzmye B and IL-13 concentrations in individual patients [47] with granzyme B displaying cytotoxic potential [47].

Thus, based on these features there is reason to speculate that the pathogenetic concepts behind asthma and EoE might be similar. Recent overviews in EoE have focused on the effector part with T-helper cells as major producers of IL-9, IL-13 and IL-5, as well as eotaxin, which promote eosinophil and mast cell accumulation and activation, both of which produce TGF- $\beta$ leading to remodeling and structural changes of esophageal smooth muscle [9].

In asthma, similar processes are present. In addition, release of neurotrophins as well as allergen-dependent accumulation of dendritic cells play a role in the immunopathogenesis of asthma where cytotoxic phenomena might also be operating. It has, therefore, been speculated that chronic allergic asthma as well as intrinsic asthma might be associated with cell-mediated autoimmunity. Whether similar mechanisms play a role in EoE requires further investigation.

In summary, there are many similarities between asthma and EoE which go beyond the regulation of eosinophilia. Findings derived from one of these diseases should stimulate similar investigations in the other to further dissect or combine the pathogenesis of these two diseases. Understanding why eosinophilic inflammation in one case manifests itself in the esophagus and in another in the airways, and occasionally in both, remains a fascinating conundrum. Resolving any of the questions in one of these diseases will certainly facilitate our understanding of the other.

\section{Disclosure Statement}

J.C.V. declares that no financial or other conflict of interest exists in relation to the content of the article.
58

Dig Dis 2014;32:54-60

DOI: $10.1159 / 000357010$
Virchow 


\section{References}

$>1$ Kanakala V, Lamb CA, Haigh C, Stirling RW, 16 Dellon ES, Chen X, Miller CR, Woosley JT, Attwood SE: The diagnosis of primary eosinophilic oesophagitis in adults: missed or misinterpreted? Eur J Gastroenterol Hepatol 2010;22:848-855

$\checkmark 2$ Pasha SF, Sharma VK, Crowell MD: Current concepts and treatment options in eosinophilic esophagitis. Curr Opin Investig Drugs 2006;7:992-996.

$>3$ Lockett GA, Holloway JW: Genome-wide as- 18 sociation studies in asthma; perhaps, the end of the beginning. Curr Opin Allergy Clin Immunol 2013;13:463-469.

4 Sherrill JD, Gao PS, Stucke EM, et al: Variants of thymic stromal lymphopoietin and its receptor associate with eosinophilic esophagitis. J Allergy Clin Immunol 2010;126:160 165.e3.

5 Blanchard C, Wang N, Stringer KF, et al: Eotaxin-3 and a uniquely conserved gene-expression profile in eosinophilic esophagitis. J Clin Invest 2006;116:536-547.

-6 Spergel JM: New genetic links in eosinophilic esophagitis. Genome Med 2010;2:60.

$>7$ Wlasiuk G, Vercelli D: The farm effect, or: when, what and how a farming environment protects from asthma and allergic disease. Curr Opin Allergy Clin Immunol 2012;12: 461-466.

$>8$ von Mutius E: Maternal farm exposure/ingestion of unpasteurized cow's milk and allergic disease. Curr Opin Gastroenterol 2012;28 570-576.

$\checkmark 9$ Abonia JP, Rothenberg ME: Eosinophilic esophagitis: rapidly advancing insights. Annu Rev Med 2012;63:421-434.

10 Covar RA, Szefler SJ, Zeiger RS, et al: Factors associated with asthma exacerbations during a long-term clinical trial of controller medications in children. J Allergy Clin Immunol 2008;122:741-747.e4.

-11 Straumann A, Aceves SS, Blanchard C, et al: Pediatric and adult eosinophilic esophagitis: similarities and differences. Allergy 2012;67: 477-490.

$>12$ Chehade M, Aceves SS: Food allergy and eosinophilic esophagitis. Curr Opin Allergy Clin Immunol 2010;10:231-237.

-13 Liccardi G, Cazzola M, Walter Canonica G, Passalacqua G, D'Amato G: New insights in allergen avoidance measures for mite and pet sensitized patients. A critical appraisal. Respir Med 2005;99:1363-1376.

-14 Virchow JC Jr, Kroegel C, Walker C, Matthys $\mathrm{H}$ : Inflammatory determinants of asthma severity: mediator and cellular changes in bronchoalveolar lavage fluid of patients with severe asthma. J Allergy Clin Immunol 1996; 98:S27-S33, discussion S33-S40.

15 Filley WV, Holley KE, Kephart GM, Gleich GJ: Identification by immunofluorescence of eosinophil granule major basic protein in lung tissues of patients with bronchial asthma. Lancet 1982;2:11-16. Shaheen NJ: Diagnostic utility of major basic protein, eotaxin-3, and leukotriene enzyme staining in eosinophilic esophagitis. Am J Gastroenterol 2012;107:1503-1511.

7 Virchow JC Jr, Holscher U, Virchow C Sr: Sputum ECP levels correlate with parameters of airflow obstruction. Am Rev Respir Dis 1992;146:604-606

8 Virchow JC Jr, Oehling A, Boer L, et al: Pulmonary function, activated $\mathrm{T}$ cells, peripheral blood eosinophilia, and serum activity for eosinophil survival in vitro: a longitudinal study in bronchial asthma. J Allergy Clin Immunol 1994;94:240-249.

19 Amin K: The role of mast cells in allergic inflammation. Respir Med 2012;106:9-14

20 Abonia JP, Blanchard C, Butz BB, et al: Involvement of mast cells in eosinophilic esophagitis. J Allergy Clin Immunol 2010;126:140 149.

21 Dougherty RH, Sidhu SS, Raman K, et al: Accumulation of intraepithelial mast cells with a unique protease phenotype in $\mathrm{T}(\mathrm{H}) 2$-high asthma. J Allergy Clin Immunol 2010;125: 1046-1053.e8.

22 Aceves SS, Chen D, Newbury RO, Dohil R, Bastian JF, Broide DH: Mast cells infiltrate the esophageal smooth muscle in patients with eosinophilic esophagitis, express TGF- $\beta 1$, and increase esophageal smooth muscle contraction. J Allergy Clin Immunol 2010;126: 1198-1204.e4.

23 Lucendo AJ, Arias A, De Rezende LC, et al: Subepithelial collagen deposition, profibrogenic cytokine gene expression, and changes after prolonged fluticasone propionate treatment in adult eosinophilic esophagitis: a prospective study. J Allergy Clin Immunol 2011; 128:1037-1046.

24 Redington AE, Madden J, Frew AJ, et al: Transforming growth factor-beta 1 in asthma. Measurement in bronchoalveolar lavage fluid. Am J Respir Crit Care Med 1997;156:642647.

25 Firszt R, Francisco D, Church TD, Thomas JM, Ingram JL, Kraft M: Interleukin-13 induces collagen type-1 expression through matrix metalloproteinase-2 and transforming growth factor- $\beta 1$ in airway fibroblasts in asthma. Eur Respir J 2013, E-pub ahead of print.

26 Cohen MC, Rao P, Thomson M, Al-Adnani M: Eosinophils in the oesophageal mucosa: clinical, pathological and epidemiological relevance in children: a cohort study. BMJ Open 2012;2:e000493.

27 Humbert M, Berger W, Rapatz G, Turk F: Add-on omalizumab improves day-to-day symptoms in inadequately controlled severe persistent allergic asthma. Allergy 2008;63: 592-596.

28 Rocha R, Vitor AB, Trindade E, et al: Omalizumab in the treatment of eosinophilic esophagitis and food allergy. Eur J Pediatr 2011;170: 1471-1474.
29 Lommatzsch M, Korn S, Buhl R, Virchow JC: Against all odds: anti-IgE for intrinsic asthma? Thorax 2013, E-pub ahead of print.

30 Kroegel C, Julius P, Matthys H, Virchow JC Jr, Luttmann W: Endobronchial secretion of interleukin-13 following local allergen challenge in atopic asthma: relationship to interleukin-4 and eosinophil counts. Eur Respir J 1996;9:899-904.

31 Corren J, Lemanske RF, Hanania NA, et al: Lebrikizumab treatment in adults with asthma. N Engl J Med 2011;365:1088-1098.

32 Blanchard C, Mingler MK, Vicario M, et al: IL-13 involvement in eosinophilic esophagitis: transcriptome analysis and reversibility with glucocorticoids. J Allergy Clin Immunol 2007;120:1292-1300.

33 Blanchard C, Stucke EM, Burwinkel K, et al: Coordinate interaction between IL-13 and epithelial differentiation cluster genes in eosinophilic esophagitis. J Immunol 2010;184: 4033-4041.

34 Niranjan R, Rayapudi M, Mishra A, Dutt P, Dynda S: Pathogenesis of allergen-induced eosinophilic esophagitis is independent of interleukin (IL)-13. Immunol Cell Biol 2013;91: 408-415.

35 Leckie MJ, ten Brinke A, Khan J, et al: Effects of an interleukin-5 blocking monoclonal antibody on eosinophils, airway hyper-responsiveness, and the late asthmatic response. Lancet 2000;356:2144-2148.

-36 Straumann A, Conus S, Grzonka P, et al: Anti-interleukin-5 antibody treatment (mepolizumab) in active eosinophilic oesophagitis: a randomised, placebo-controlled, doubleblind trial. Gut 2010;59:21-30.

37 Pavord ID, Korn S, Howarth P, et al: Mepolizumab for severe eosinophilic asthma (DREAM): a multicentre, double-blind, placebo-controlled trial. Lancet 2012;380:651659.

38 Castro M, Mathur S, Hargreave F, et al: Reslizumab for poorly controlled, eosinophilic asthma: a randomized, placebo-controlled study. Am J Respir Crit Care Med 2011;184: 1125-1132.

39 McGrath KW, Icitovic N, Boushey HA, et al: A large subgroup of mild-to-moderate asth$\mathrm{ma}$ is persistently noneosinophilic. Am J Respir Crit Care Med 2012;185:612-619.

40 Agache I, Akdis C, Jutel M, Virchow JC: Untangling asthma phenotypes and endotypes. Allergy 2012;67:835-846.

41 Walker C, Bode E, Boer L, Hansel TT, Blaser $\mathrm{K}$, Virchow JC Jr: Allergic and nonallergic asthmatics have distinct patterns of T-cell activation and cytokine production in peripheral blood and bronchoalveolar lavage. Am Rev Respir Dis 1992;146:109-115.

-42 Bratke K, Lommatzsch M, Julius P, et al: Dendritic cell subsets in human bronchoalveolar lavage fluid after segmental allergen challenge. Thorax 2007;62:168-175. 
43 Lommatzsch M, Virchow JC: The neural underpinnings of asthma. J Allergy Clin Immunol 2007;119:254-255, author reply 255 .

-44 Virchow JC, Julius P, Lommatzsch M, Luttmann W, Renz H, Braun A: Neurotrophins are increased in bronchoalveolar lavage fluid after segmental allergen provocation. Am J Respir Crit Care Med 1998;158:2002-2005.
45 Nassenstein C, Braun A, Erpenbeck VJ, et al: The neurotrophins nerve growth factor, brain-derived neurotrophic factor, neurotrophin-3, and neurotrophin-4 are survival and activation factors for eosinophils in patients with allergic bronchial asthma. J Exp Med 2003;198:455-467.

46 Bratke K, Bottcher B, Leeder K, et al: Increase in granzyme $\mathrm{B}+$ lymphocytes and soluble granzyme B in bronchoalveolar lavage of allergen challenged patients with atopic asthma. Clin Exp Immunol 2004;136:542-548.
7 Tschopp CM, Spiegl N, Didichenko S, et al: Granzyme B, a novel mediator of allergic inflammation: its induction and release in blood basophils and human asthma. Blood 2006; 108:2290-2299.

48 Virchow JC Jr: Intrinsic asthma; in Busse WW, Holgate ST (eds): Asthma and Rhinitis. Oxford, Blackwell, 2000. 